\title{
Symmetrical Peripheral Gangrene complicating Staphylococcal Toxic Shock Syndrome
}

\author{
Col SP Rai", LS Neog+, Col D Bhattacharyya", Lt Col M Ganguli**
}

MJAFI 2008; 64 : 181-182

Key Words: Symmetrical peripheral gangrene; Toxic shock syndrome

\section{Introduction}

Sudden onset symmetrical peripheral gangrene (SPG) $\checkmark$ is a relatively uncommon clinical entity which was initially described in 1981 by Hutchison. It is defined as symmetric distal ischemic damage in two or more sites in the absence of major vascular occlusive disease [1$3]$. It is a manifestation of many systemic disorders and accompanies infectious disease of various etiologies, shock, drugs and malignancy [1-3].

Toxic shock syndrome (TSS) is an acute life threatening inflammatory response characterized by fever, rash, hypotension, constitutional symptoms and multiorgan involvement $[4,5]$. The disease first described in 1978, is typically associated with tampon use in healthy menstruating women. The disease also exists in men, neonates and non-menstruating women. TSS is a toxin mediated disease caused by coagulase - positive staphylococci (S aureus) and group A beta- hemolytic streptococci (S pyogenes) [4,5]. We report a case of staphylococcal TSS, complicated by rapid onset SPG involving the toes and fingers.

\section{Case Report}

A 23 year old female presented with complaints of pain and swelling right breast with moderate grade continuous fever for seven days, obtundation of sensorium with pain and bilaterally symmetrical bluish discolouration of all the toes and fingers for three days. She was married, had two issues and was breast-feeding the younger child. She had undergone induced abortion two months earlier and had one normal menstruation after that. There was no history of drug intake prior to onset of illness.

Physical examination revealed an ill looking, drowsy and disoriented young woman. Her pulse was 98/ minute, blood pressure of $90 / 66 \mathrm{~mm} \mathrm{Hg}$, respiratory rate of 24/minute and temperature of $99^{\circ} \mathrm{F}$. She had pallor, icterus and bilateral conjunctival congestion with subconjunctival haemorrhage. She had desquamation of skin around eyes, mouth, genitalia, breast, palms and soles (Fig.1). Crustation and ulceration were present in mouth and genitals. Purpuric spots were seen all over the body. Distal digits showed gangrenous changes with ecchymosis in both hands and feet. Pulse in both radial, ulnar and dorsalis pedis arteries were normal. $4 \times 5 \mathrm{~cm}$ size, tender irregular lump was seen in outer upper quadrant of right breast along with fissuring of right nipple. Other systems were normal.

On investigation haemoglobin was $10.8 \mathrm{gm} / \mathrm{dl}$, total leucocyte count $17,400 / \mathrm{mm}^{3}$, polymorphs $80 \%$, lymphocytes $10 \%$, monocytes $8 \%$, eosinophils $2 \%$ and platelet count $28000 / \mathrm{mm}^{3}$. Peripheral blood smear showed normocytic, normochromic red cells, polymorphonuclear leucocytosis and thrombocytopenia. There were no toxic granules. Chest radiograph and electrocardiogram were unremarkable. Blood urea was $181 \mathrm{mg} / \mathrm{dl}$, serum creatinine $3.3 \mathrm{mg} / \mathrm{dl}$, serum bilirubin $3.2 \mathrm{mg} / \mathrm{dl}$, aspartate aminotransferase $246 \mathrm{IU} / \mathrm{L}$ and alanine aminotransferase of $122 \mathrm{IU} / \mathrm{L}$. Her coagulation profile and serum electrolytes were normal. Stool examination showed 10-12 red blood cells and 4-6 pus cells/ HPF. Stool culture grew lactose fermenter. Culture from fine needle aspirate from breast lump and blood culture grew staphylococcus aureus. Urine and high vaginal swab cultures grew $E$ coli.

She was managed with Inj clindamycin $600 \mathrm{mg}$ intravenous (IV) eight hourly and Inj targocid $400 \mathrm{mg}$ IV 12 hourly for two weeks along with pantoprazole, blood transfusions, betadine application over abraded area, mouth wash and vaginal douche. She improved and became afebrile in three weeks and her breast lump regressed. Her renal functions and liver functions improved and platelet count increased to $95000 /$ $\mathrm{mm}^{3}$ in five days. A clear line of demarcation of dry gangrene appeared (Fig.2) and she was subjected to excision of toes and tips of fingers along with reconstruction after one month.

\section{Discussion}

Only sporadic reports of SPG have appeared until

*Senior Advisor (Medicine \& Respiratory Medicine), Military Hospital (CTC) Pune - 4. ${ }^{+}$Consultant Dermatologist, Apollo Hospital, Delhi. \#Senior Advisor (Medicine \& Respiratory Medicine), Army Hospital R\&R, Delhi Cantt - $10 .{ }^{* *}$ Classified Specialist (Pathology), Military Hospital Namkum, Ranchi - 10. 


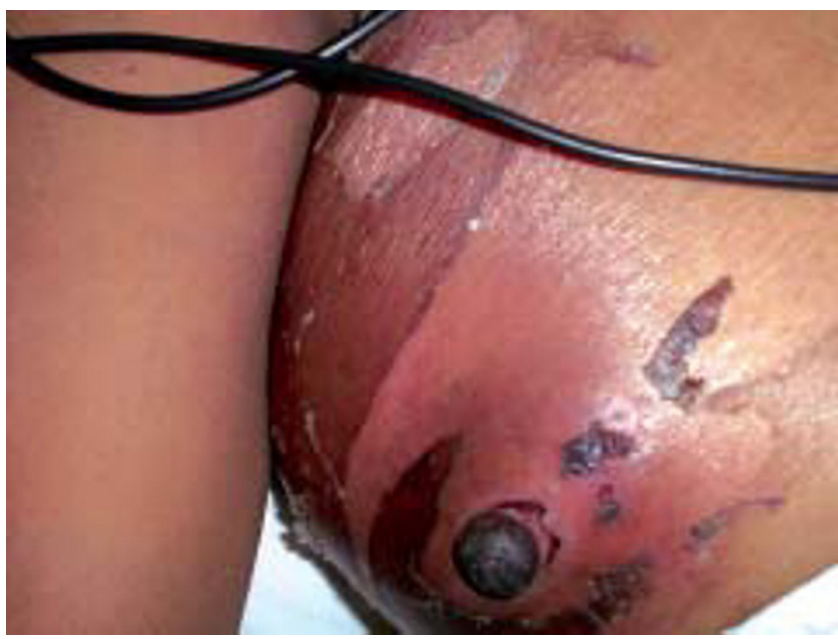

Fig. 1 : Photograph showing cracked nipple, breast abscess with surrounding oedema and desquamation of skin.

now, but there has been no report of a case complicating TSS. TSS affects due to staphylococcal endotoxins (TSS Toxin-1), exfoliative toxins or enterotoxins. The disease is characterized by fever, rash, desquamation, circulatory shock and multisystem involvement $[4,5]$. TSS has been linked to many bacterial infections, including pneumonia, osteomyelitis, sinusitis, skin and gynecological infections.

Staphylococcal TSS is more common in women and the mortality rate is $2.5 \%$ for menstrual cases and $6.4 \%$ for non-menstrual cases. Our patient had a cracked nipple and possibly during breast feeding the colonized staphylococci gained access to deeper tissue leading to breast abscess. She had features of TSS in form of fever, altered consciousness, hypotension, desquamation, mucosal inflammation, thrombocytopenia with deranged renal and hepatic functions. TSS should be differentiated from erythema multiforme, drug reaction, kawasaki disease and staphylococcal scalded skin syndrome. Treatment of TSS includes drainage of the site of toxin production, aggressive fluid resuscitation and administration of anti-staphylococcal antibiotics. Electrolyte abnormalities must be corrected and organ failures must be managed conservatively. Patients with severe illness should be treated with IV immunoglobulin $400 \mathrm{mg} / \mathrm{kg}$ once, which contains high levels of neutralizing antibody to TSS toxins.

The most common cause of SPG in the clinical setting is septicemia. Other conditions include disseminated intravascular coagulation (DIC), shock, drugs and malignancy [1-3]. SPG may also be a manifestation of multiorgan system failure. Infections which can cause SPG are meningococcus, streptococcus, pneumococcus, staphylococcus, E coli, pseudomonas, S paratyphi, klebsiella, DF-2 Gram negative bacillus, viral gastroenteritis, varicella and rubeola. It has also been reported in plasmodium falciparum infection [2]. Drugs

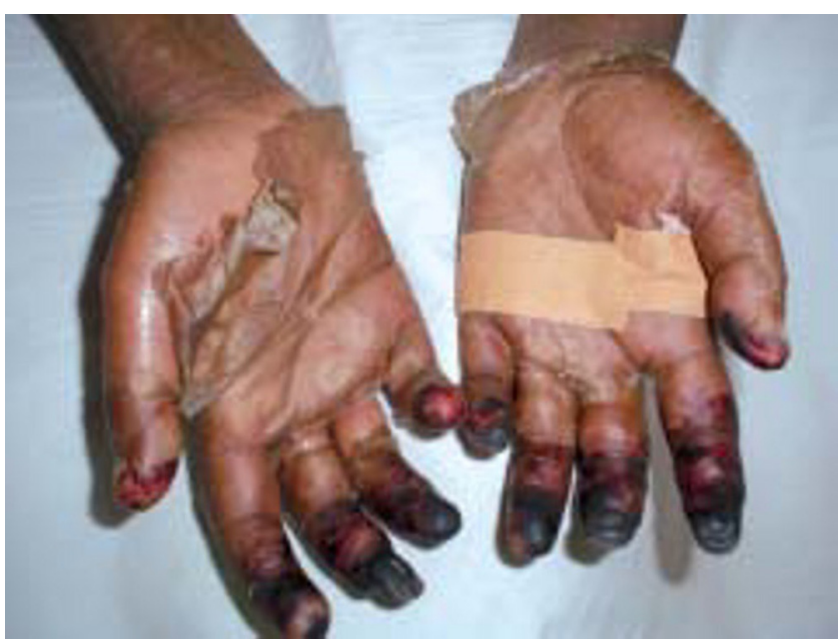

Fig. 3 : Photographs showing dry gangrene of fingers with clearcut demarcation and desquamation of skin.

like adrenaline, nor-adrenaline and dopamine are known to cause SPG [3].

SPG in our patient started with cyanosis of fingers and toes, followed by gangrene. There were no manifestations of DIC. Clearcut demarcation of gangrene was detected on the $10^{\text {th }}$ day. The natural history of SPG has many similarities with severe cold injury. In both conditions there is dry gangrene, mummification and absence of infection. The peripheral tissue injury in both should be treated with inter digital padding and protection from trauma. Since initial assessment of tissue damage may not be correct, early surgery should be resisted. Amputation and skin grafting is done after the demarcation line of dry gangrene sets in. SPG has been successfully treated with epoprostenol and tissue plasminogen activators [6].

\section{Conflicts of Interest}

None identified

\section{References}

1. Kashyap R, Behl RK, Mahajan S, Jaret P, Patial RK, Kaushal SS. Symmetrical peripheral gangrene due to viral gastroenteritis. JAPI 2004;52:500-1.

2. Kakati S, Doley B, Barman B, Anjana Devi. Symmetric peripheral gangrene and falciparum malaria. JAPI 2005; 52 : 498-9.

3. Kumar S, Alexander M, Joseph M, Gnanamuthu C. Symmetrical peripheral gangrene: association with adrenaline administration. CCA 2004; 2:19-21.

4. Deresiewicz RL. Staphylococcal toxic shock syndrome. In: Leung DYM et al, editors. Super-antigens: Molecular biology, immunology, and relevance to human disease. New York: Marcel Dekker, 1997; 435-79.

5. Salandy D, Brenner B. Toxic shock syndrome. emedicine.com 2004 [Cited 2004 Nov 16], available from http:// www.emedicine.com/EMERG/topic 600.htm.

6. Denning DW, Gilliand L, Hewlett A, et al. Peripheral symmetrical gangrene successfully treated with tissue plasminogen activator. Lancet 1986; 2:1401-2. 